\title{
Itinéraires Itinéraires
}

Littérature, textes, cultures

2019-2 et $3 \mid 2019$

Corps masculins et nation : textes, images, représentations

\section{L'horreur du féminin dans le cycle des Jeunes Filles d'Henry de Montherlant, ou comment on émascule la France}

The Horrors of Femininity in the Anthology of Les Jeunes Filles by Henry de Montherlant, or How to Emasculate France

Pierre Damamme

\section{(2) OpenEdition}

Journals

Édition électronique

URL : http://journals.openedition.org/itineraires/6656

DOI : $10.4000 /$ itineraires.6656

ISSN : 2427-920X

Éditeur

Pléiade

Référence électronique

Pierre Damamme, «L'horreur du féminin dans le cycle des Jeunes Filles d'Henry de Montherlant, ou comment on émascule la France », Itinéraires [En ligne], 2019-2 et 3 | 2019, mis en ligne le 11 décembre 2019, consulté le 15 décembre 2019. URL : http://journals.openedition.org/itineraires/6656 : DOI : 10.4000/itineraires.6656

Ce document a été généré automatiquement le 15 décembre 2019.

\section{(ब) $\Theta \Theta$}

Itinéraires est mis à disposition selon les termes de la licence Creative Commons Attribution - Pas d'Utilisation Commerciale - Pas de Modification 4.0 International. 


\title{
L'horreur du féminin dans le cycle des Jeunes Filles d'Henry de Montherlant, ou comment on émascule la France
}

\author{
The Horrors of Femininity in the Anthology of Les Jeunes Filles by Henry de \\ Montherlant, or How to Emasculate France
}

Pierre Damamme

1 Pour mesurer le tollé provoqué par le cycle des Jeunes Filles d'Henry de Montherlant paru entre 1936 et 1939, on peut se reporter à la presse de l'époque. Les chrétiens ne goûtent guère le roman à l'image du R. P. Dérély, promoteur des Cadettes du Christ, cherchant à défendre «l'honneur » des jeunes filles de France après lecture de ce livre «ignoble» et "odieux", écrit par un auteur "qui ne respecte rien, ni Dieu, ni la religion, ni la famille, ni la femme, ni l'amour, ni ses lecteurs, ni lui-même » (cité dans Sipriot 1979: 159). La condamnation est claire et ne laisse aucune place au débat: Montherlant est jugé coupable. Dans Les Nouvelles littéraires (août 1936) s'ouvre une enquête menée par plusieurs lectrices afin de savoir si les portraits de femmes sont conformes à la réalité. Certaines s'insurgent contre les monstrueuses créations littéraires de l'écrivain tandis que d'autres prennent sa défense, reconnaissant avoir déjà croisé dans leur vie des "Andrée Hacquebaut » ou des "Solange Dandillot ", les deux personnages féminins principaux du roman. Un nouveau pas est franchi en 1949 par Simone de Beauvoir dans un violent réquisitoire intitulé «Montherlant ou le pain du dégoût » qui dresse le portrait d'un Montherlant lâche, maltraitant les femmes pour mieux éprouver son illusoire domination. Pour elle, l'écrivain contribue à perpétuer le mythe de la Femme, reconduisant la croyance en cette "mystérieuse essence »: la féminité (Beauvoir [1949] 2008b : 558).

2 Dépeint en ennemi des femmes depuis la parution du premier tome des Jeunes Filles, Montherlant tire aussi profit de cette image, par exemple en donnant à la deuxième édition de La Reine morte en 1942 un sous-titre provocateur : «Comment on tue les 
femmes ». Sans fournir un tel mode d'emploi, le cycle des Jeunes Filles souligne déjà le risque d'une France fragilisée par une contagion du «féminin ». Dans ce roman semiépistolaire, Montherlant met en effet en scène plusieurs femmes amoureuses du héros, Pierre Costals, qui apparaissent comme autant d'êtres faibles, dépendants et entièrement soumis à leur passion.

3 À la fin du dernier volume, l'auteur énumère les maux qu'il juge caractéristiques de l'occident moderne -irréalisme, dolorisme, vouloir-plaire, grégarisme et sentimentalisme - qui, réunis, forment ce qu'il appelle dans d'autres ouvrages la "morale de midinette ». À l'origine d'un avilissement de la France, cette morale qui "se réduit presque uniquement à être "bon", [que dis-je], à être "gentil", à être aimable, à être facile" (Montherlant [1938] 1963: 843) provoque chez l'écrivain un sursaut héroïque destiné à conjurer ce qui prend la forme métaphorique d'une "émasculation ". Ce thème de l'émasculation hante les consciences de l'époque depuis la fin de la Grande Guerre. En effet, la guerre a pu être vécue par bon nombre de combattants comme un moment d'impuissance. Les témoins de la guerre décrivent un déchaînement de feu sans pareil qui entraîne le massacre des hommes et des bêtes et la destruction des villages. Pendant ces années de guerre, il faut apprendre à vivre au milieu des paysages dévastés et des corps déchiquetés.

Pourtant, à la fin de la guerre, le mythe viril ne s'effondre pas mais se transforme comme l'explique Stéphane Audouin-Rouzeau (2011: 405): «c'est d'une virilité douloureuse qu'il s'agit désormais, mais intacte, telle que la répliqueront d'ailleurs tant de monuments aux morts ». Les blessures des combattants sont autant de preuves de leur courage et les décorations sont arborées avec fierté. Même si la guerre pouvait apparaître comme une boucherie (comme tant d'écrivains l'ont noté), elle reste pour Montherlant un motif puissant d'exaltation. Ainsi, ses biographes rapportent que celuici aurait continué à porter son uniforme après la fin de la guerre pendant plusieurs mois, signe de son attachement à cette période de camaraderie virile.

Le retour à la vie bourgeoise marque dès lors la fin de l'âge viril et l'entrée dans un monde qui peut sembler dépourvu de grandeur aux hommes qui risquèrent leur vie à la guerre. Le temps de l'aventure et de la vie intense s'achève sans rien pour ranimer la ferveur et l'ivresse des combats. Or si la guerre était un monde d'hommes, la paix semble aux yeux de certains anciens combattants favoriser les femmes. Ainsi, Drieu la Rochelle fait par exemple dans Gilles la peinture d'hommes sans influence et sans repères, obligés de s'adapter aux exigences d'une société faite pour les femmes :

Les femmes sont dans la société jusqu'au cou. Et la société a été faite pour elles, en rapport avec leurs exigences. Elles ont besoin du confort pour leurs enfants et $d u$ luxe pour leur beauté. En conséquence, les hommes comme eux [Cyrille et Gilles] n'existent pas. Ils sont sans argent, sans pouvoir. (Drieu la Rochelle [1939] 1973: 402)

Tout se passe comme si l'homme se retrouvait piégé, pris dans l'étau d'une société entièrement adaptée aux femmes et dans laquelle il lui serait impossible non seulement de trouver sa place mais même d'" exister ", comme le suggère le chiasme de la première phrase qui l'exclut définitivement.

6 Les déclarations de Montherlant à propos des Jeunes Filles orientent la lecture du roman dans ce sens. S'il présente sa tétralogie comme « une longue offensive contre l'échelle des valeurs au nom de laquelle on émascule la France » (Montherlant [1938] 1963 : 843), c'est qu'il a l'impression, comme Drieu la Rochelle, de vivre un moment d'apothéose féminine. Ainsi, en stigmatisant les femmes, il espère peut-être d'abord restaurer une 
virilité qu'il juge menacée. Il semble alors faire de la virilité et de la féminité des essences intangibles au lieu de les envisager comme des constructions sociales et culturelles comme le lui reproche Simone de Beauvoir.

Pour autant, il n'est pas évident que l'auteur ne fasse dans ce roman qu'exprimer son dégoût pour les femmes. En effet, il dénonce moins les femmes qu'un certain idéal de la femme qu'il entend démystifier et qui correspond à une réalité d'époque. Il se livre alors à une satire d'une grande férocité littéraire, et ce, au nom d'un idéal viril qui repose principalement sur la puissance sexuelle prêtée au mâle. S'il continue ainsi d'opposer le masculin au féminin, il n'en ménage pas moins une place pour les femmes. En effet, au lieu d'y voir une simple galerie de clichés misogynes, l'on pourrait considérer que le roman se veut avant tout un jeu avec les stéréotypes d'une époque.

\section{La démystification de l'« éternel féminin »}

8 La critique s'est attachée à montrer la place subalterne qu'occupent en général les femmes dans l'œuvre de Henry de Montherlant. Paule d'Arx (1995: 76) évoque ainsi son "antiféminisme incurable». Les explications de cette défiance envers les femmes seraient multiples, qu'on avance les rapports conflictuels que l'auteur entretint avec sa mère durant l'enfance et l'adolescence (Blanc 1995 : 56) ou un désir homosexuel plus ou moins refoulé (Kremer 1987: 38). Dans son réquisitoire intégré au Deuxième Sexe, Simone de Beauvoir cherche à démontrer la misogynie de Montherlant. Selon elle, ce dernier refuse l'épreuve du réel par lâcheté et ainsi «se rêve une subjectivité souverainement repliée sur soi-même" (Beauvoir [1949] 2008a: 251). Sa haine des femmes s'expliquerait par une impossible ouverture à l'autre. Aussi les considère-t-il comme faibles et passives pour mieux éprouver sa supériorité : "il ne s'agit pas d'établir avec la femme aucun rapport intersubjectif : elle ne doit être au royaume de l'homme qu'un simple objet animé ; jamais on ne l'envisagera comme sujet » (Beauvoir [1949] 2008a : 256).

\section{Une peinture d'époque}

9 La femme apparaît d'abord dans le roman comme un motif de dégoût: "Femmes, toujours malades, toujours malsaines, jamais tout à fait nettes ", constate Costals dans son journal intime comme s'il craignait une sorte de contagion (Le Démon du bien : 50). Le vocabulaire médical est fréquemment employé : les lettres des femmes sont ainsi soumises à une "quarantaine » par le héros du roman; le mot «amour » mérite une «désinfection» (Montherlant [1957] 1963 : 1323) ; les femmes sont des «lépreuses » comme l'indique le titre du quatrième volume; elles plongent l'homme dans un « climat anémiant " (Les Lépreuses : 60). Aussi la femme se présente-t-elle dans le roman comme un cas clinique à étudier pour mesurer l'affaiblissement de la nation. En somme, Montherlant pose un diagnostic à la manière d'un médecin. Le héros du roman, Costals, écrivain lui-même, apparaît à cet égard comme un double de l'auteur : tour à tour présenté comme médecin, savant, entomologiste, chimiste, son rôle est avant tout d'observer les jeunes filles: "Je vous suis avec intérêt dans vos différents états ", répond-il de manière apparemment neutre à Andrée (Pitié pour les femmes : 182).

Dans sa peinture des femmes, Montherlant reprend toutes les croyances stéréotypées qui pèsent sur elles. Le psychologue social Baron Byrne a fait la liste des défauts 
généralement prêtés aux femmes et l'on pourrait sans peine les appliquer aux personnages féminins de Montherlant qui peinent à exister de manière autonome. En effet, les femmes y apparaissent bien «soumises, dépendantes, pleines de tact, douces, bavardes, passives, plus enclines à suivre qu'à diriger, peu sûres d'elles-mêmes, dépourvues d'ambition, sensibles aux sentiments d'autrui, trop émotives " (Amossy 1991 : 170). Néanmoins, si ces clichés sont repris par Montherlant, c'est aussi parce qu'ils sont l'expression d'une époque. Les héroïnes apparaissent comme des caricatures car la société de l'entre-deux-guerres ne laisse pas d'autres figures possibles aux femmes. Montherlant souhaitait avant tout conformer ses portraits à la réalité de la société de son temps. Le réalisme est en effet pour lui une valeur clé au point qu'il se décrit par exemple comme un simple photographe, un "photographe d'âmes" (Montherlant [1920] 1963 : 42). C'est pourquoi il n'est pas indifférent non plus dans son roman aux conditions sociales qui empêchent la femme de s'émanciper.

11 Même s'il ne remonte pas aux causes de la domination masculine - ce que lui reproche Simone de Beauvoir - il n'en montre pas moins que les possibilités d'exister pour la femme sont minimes: "Vous ne voyez pas clair? Eh! Qui vous aurait appris à voir clair? Toute l'éducation des filles est faussée ", affirme Costals dans une ultime lettre à Andrée (Les Lépreuses : 213). Montherlant reprendra dans une note de ses carnets cette critique d'une éducation coercitive, notamment à l'égard des femmes :

Garçons et filles, grand tort de les élever pour la société, non pour eux-mêmes. On ne cherche pas à développer des individualités fortes; on préfère des êtres dociles et qui ont des besoins par lesquels on les tient. Cela est sensible surtout dans l'éducation des filles. (Monique, Françoise). (Montherlant [1957] 1963 : 104)

Dans Les Jeunes Filles, Montherlant met par exemple en cause les magazines féminins comme un facteur d'aliénation. La femme peut-elle être autre chose qu'un être grégaire si elle se contente de lire "ces articles rigolards où, à la page féminine des hebdomadaires, Sylphide ou Cousine Annie indique à ses "sœurs" les recettes pour "se faire une personnalité" »? (Les Lépreuses: 78). L'ironie apparaît dans l'usage des guillemets qui mettent à distance l'objectif paradoxal de ces magazines censés permettre à chaque femme de devenir unique en indiquant à toutes la même recette. Montherlant renvoie ainsi à une réalité d'époque car ces magazines connaissent une popularité grandissante depuis les années 1930 comme le rappelle la philosophe Françoise Collin, qui souligne également leur rôle infantilisant dans la mesure où ils "maintiennent l'attrait pour une beauté superficielle» et incitent les femmes «à maitriser leur situation, mais pas à la remettre en question" (Collin 1992: 489). En mettant en cause les magazines féminins, Montherlant donne ainsi une cause sociale au grégarisme qu'il prête aux femmes, souvent représentées dans le roman comme un «troupeau » aux réactions prévisibles.

\section{La férocité littéraire}

13 La férocité dont Montherlant fait preuve à l'égard des femmes répond en réalité à un objectif clair : démystifier un certain idéal de la femme, celle de la "Sphynge éternelle " (Le Démon du bien : 88), « Ève mystérieuse » au " sourire ensorceleur » (Les Jeunes Filles : 30), autant d'expressions fallacieuses pour l'auteur. La perspective est volontiers cynique, au sens d'abord philosophique du mot, dans la mesure où Montherlant s'efforce de démasquer les conventions et les clichés. Comme l'a remarqué Jean-François Domenget, la posture de Montherlant rejoint celle du philosophe cynique de l'Antiquité qui refuse 
d'être « dupe de la comédie humaine et [qui], comme le chien, aboie contre la société de son temps » (Domenget $2014: 57$ ). C'est pourquoi les femmes sont dans le roman l'objet d'une violente satire. Or la satire, à la différence du comique, implique « une axiologie affichée et tenue pour supérieure" (Moura 2010: 87), une visée correctrice qui présuppose un système de valeurs. Comme les cyniques grecs, Montherlant invoque la nature pour condamner les artifices qui lui semblent caractériser l'univers féminin. Il reproche aux femmes d'avoir oublié leur nature originelle, de s'être réglées sur les normes corrompues de la société. Montherlant ne se fixe pas d'autre rôle que celui d'" [arracher] l'enveloppe brillante dont chacun, paradant sous les regards, recouvrait sa laideur intérieure ", selon la formule d'Horace (Moura 2010 : 87). Ainsi, les notations cruelles abondent dans ce roman, y compris au sens étymologique de cruor qui désigne la chair mise à nu, à vif. Montherlant décrit par exemple, pendant un acte d'amour, les lèvres entr'ouvertes de Solange, «montrant les dents petites, comme on voit dans les étals de boucherie aux têtes décollées des moutons" (Pitié pour les femmes: 124). L'image de la décapitation donne au rapport amoureux une valeur funèbre comme si la pulsion de vie (Eros) appelait en retour la pulsion de mort (Thanatos).

Montherlant ne combat pas seulement la femme sur un front idéologique, mais également sur un front littéraire. Désireux de coller au réel, il cherche à émanciper son texte des codes habituels du roman; il construit un roman qui, paradoxalement, désavoue le romanesque, d'où son extrême liberté de ton et d'allure, sa résistance par exemple aux belles scènes romantiques, son refus des descriptions attendues ou du lyrisme. Les interventions de l'auteur brisent ainsi fréquemment l'illusion romanesque: "De tout temps, les romanciers ont fait des phrases sur le décor où se rencontrent leurs amoureux" (Les Jeunes Filles: 84), "ce chapitre est une sorte de galéjade " (Les Jeunes Filles: 168), «la "psychologie" qu'un romancier met dans ses bouquins, on sait ce que c'est: de $a$ à $z$; du trompe-l'œil » (Les Lépreuses : 40). Ainsi, même le roman n'échappe pas à la force dissolvante du cynisme de Montherlant qui invite son lecteur à rester lucide sur le récit des égarements amoureux de ses personnages.

15 Aussi féroce soit-elle, la satire des femmes est peut-être un moyen d'inciter ces dernières à se révolter contre l'ordre d'une société qui les voue à cultiver l'artifice. L'apologue qui clôt le cycle des Jeunes Filles ne laisse aucun doute à cet égard. Le vieux maître de classe qui fait venir l'élève qu'il a tant brimé lui avoue à la fin son attachement. Montherlant, comme le maître de classe, convoque à la fin ses lectrices tant brusquées, tant malmenées, pour leur avouer qu'il ne cherche que leur bien. Ce roman fondé sur la provocation attaque donc pour mieux inviter ses lectrices à se défendre, comme le suggère Costals : «Moi, mon rôle est d'attaquer, mais enfin, que la société se défende! Or, si je cherche à conquérir les corps, ou si je cherche à troubler les esprits et les âmes, c'est toujours la même chose : pas de défense ! À perpétuité le fromage mou. Je joue mon jeu; eux, ils ne jouent pas le leur» (Pitié pour les femmes: 144). Ce constat s'applique à la nation, «fromage mou» depuis la fin de la guerre. Le lexique guerrier (attaquer, se défendre, conquérir, défense) dit l'attachement de Montherlant à une nation forte et combative, capable de prendre les armes.

16 C'est pourquoi Montherlant a pu considérer son roman comme « une œuvre salubre pour les femmes, en leur montrant ce qu'elles sont, et ce qu'est l'homme quand il ne feint pas avec elles» (Marchand 1980: 99), comme un « cours de conduite " profitable aux jeunes filles (Montherlant 1972 : 92). 


\section{Le mythe viril} mythe de la virilité. Dans le roman, la virilité s'exprime avant tout dans la manifestation de la puissance sexuelle, dans la libération totale des instincts qui rappelle ce que Nietzsche appelle "la grande santé ». Le sperme devient son emblème de sorte que Costals sent que « c'était sa destinée à lui de projeter le liquide mâle, la plus pure des substances sécrétées par les organes humains - la seule pure, - innocente et pure comme le grain de blé » (Les Lépreuses: 192). Montherlant fait de l'homme un être de désir (et non d'amour, comme la femme), doué d'un rôle fécondateur. Lui seul serait à même de régénérer la société sur un modèle physiologique et naturel. D'où la comparaison au grain de blé qui suggère que c'est au mâle que revient la mission de féconder la société. Ce qui définirait l'homme viril, ce serait donc sa capacité à jouir. Dans La Domination masculine, Pierre Bourdieu souligne la place centrale du phallus dans l'imaginaire viril, condensateur de tous les fantasmes liés à la puissance fécondante. Il montre notamment que la survalorisation de l'organe masculin est une manière de consacrer symboliquement, à la faveur d'une construction sociale et culturelle, un processus biologique: «En associant l'érection phallique à la dynamique vitale du gonflement qui est immanente à tout le processus de reproduction naturelle (germination, gestation, etc.), la construction sociale des organes sexuels enregistre et ratifie symboliquement certaines propriétés naturelles indiscutables » (Bourdieu 1998 : 27). Ainsi, Montherlant reste fidèle à la logique du système mythico-rituel analysé par Bourdieu à travers la forme paradigmatique de la société kabyle et notamment au principe d'opposition fondamental entre le passif (féminin) et l'actif (masculin). Montherlant continue à faire de l'homme le pôle essentiel de la reproduction. Le ventre de la femme se réduit alors à n'être qu'un réceptacle destiné à recevoir le phallus.

L'éloge du sperme répond vraisemblablement à une angoisse de la stérilité dans une France inquiète de sa dénatalité. Le géographe Albert Demangeon s'inquiétait par exemple en 1920 dans Le Déclin de l'Europe de la perte de vitalité et de puissance d'une Europe affaiblie et dépeuplée depuis la fin de la guerre. Contrairement à Costals qui ne doute pas de son pouvoir fécondant, le héros éponyme de Gilles de Drieu la Rochelle est ainsi hanté par le spectre d'une stérilité menaçante: «C'est la source même de la vie qui est atteinte. Plus de foutre, ou il va au bidet. Les Français n'ont plus qu'une passion, de crever... » (Drieu la Rochelle [1939] 1973 : 493). La décadence de la nation est ainsi associée à l'incapacité de produire la semence mâle. L'énergie virile s'éparpille sans rien produire.

19 Ainsi, Montherlant se livre dans le roman à une véritable démystification du «féminin » qui relève pour une part de la peinture d'une époque. Il utilise les armes de la satire pour discréditer moins peut-être les femmes qu'une certaine idolâtrie de la femme. La perspective cynique qu'il adopte lui permet de dissoudre, non sans une certaine cruauté, la nuée d'artifices qui pèse sur les femmes. Néanmoins, au lieu de dénoncer en même temps les stéréotypes virils, il les reconduit, voire les renforce à travers un éloge de la puissance sexuelle incarnée par le phallus. Il présente ainsi, à travers cette peinture des femmes, l'image d'une nation malade et grégaire, incapable de conquérir son autonomie et d'embrasser un nouvel horizon héroïque au sortir de la Grande Guerre. Pourtant, comme le remarque Romaric Sangars, il s'agit moins pour 
Montherlant «d'une confrontation des sexes que d'une confrontation de principes : le principe masculin-solaire qu'il prétendait incarner, menacé par le principe fémininlunaire lui semblant avoir polarisé son époque pour sa misère et sa dégradation " (Sangars 2012: 63). C'est pourquoi, dans le roman, il reste possible pour les femmes d'échapper, au moins en partie, au rôle de « midinette».

\section{Les identités en mouvement}

Sans faire de Montherlant un "défenseur des femmes " (c'était le titre provocant et malicieux d'une communication de Romain Lancrey-Javal lors de la « Journée Henry de Montherlant » à Bruxelles le 25 septembre 2007), l'on peut remarquer que Montherlant déjoue la binarité d'un système qui opposerait les hommes aux femmes en donnant du « jeu » à son œuvre au sens mécanique du terme : le jeu comme intervalle entre deux pièces, leur permettant de se mouvoir librement, si bien que les identités continuent à être en mouvement.

\section{La pluralité contradictoire}

21 Dans le roman, les principes masculin et féminin ne recoupent pas nécessairement l'appartenance déclarée à un sexe. Simone de Beauvoir reproche à Montherlant d'avoir " converti» la femme en "monstre» (Beauvoir [1949] 2008a: 253), mais l'écrivain permet aussi quelquefois aux femmes qu'il décrit de se définir positivement. Ainsi, il prête à Andrée certaines qualités qui en font un être à part. Le narrateur met par exemple en avant sa qualité intérieure, son intelligence et son discernement. Lisant "presque toujours des livres de valeur ", elle sait tirer bénéfice de ses lectures et, par un « sens très aigu de la valeur du temps » (Les Jeunes Filles : 58 ), elle veille à consacrer le maximum de son temps à la culture. C'est sa situation qui ne lui permet pas de s'émanciper et qui la conduit finalement à rêver sa propre vie et à s'imaginer qu'elle pourrait être aimée de Costals. La lecture est en effet le seul recours de cette provinciale condamnée aux tâches ménagères. Son destin n'eût-il pas été différent si elle avait été un homme ? Comme il renvoie étymologiquement au mâle, son prénom la situe déjà potentiellement en marge de l'univers féminin (andrê̂os désigne le masculin en grec).

Solange Dandillot - autre personnage féminin important du roman - ne se stabilise pas elle non plus complètement dans un stéréotype. Si elle est décrite comme une femmeobjet, belle et sans cervelle, elle échappe à plusieurs reprises à ce statut. Ainsi, Costals ne peut manquer de l'admirer lorsqu'elle se met à fixer le soleil sans ciller. Rare moment d'harmonie et de plénitude, cette scène décrit la mythification du couple en dieu et en déesse. Tandis que Costals se métamorphose en lion, Solange devient chatte. L'écrivain reprend l'imaginaire de la mythologie égyptienne et définit Solange comme «Celle-qui-fixe-le-Soleil » sous la forme d'un hiéroglyphe (Le Démon du bien : 188). Alors qu'elle semble se mouvoir "sur le plan surhumain ", celui des dieux, Costals est sur le point de tomber à genoux tant il est fasciné. Le mariage, dans ces conditions, devient envisageable. Cette scène est d'autant plus exceptionnelle que la femme est plus volontiers associée dans l'imaginaire montherlantien à l'immanence de la nuit qu'à la transcendance du jour. Solange semble incarner à ce stade un troisième sexe. Comme le 
remarque Costals lui-même, le prénom de la jeune femme la fait déjà participer de deux plans différents : le terrestre (Sol-) et le divin (-Ange).

Cette pluralité contradictoire suggère que les femmes peuvent échapper à des rôles prédéfinis. C'est pourquoi il n'est pas certain que Montherlant souscrive complètement aux stéréotypes que l'on peut repérer dans le roman. Comme le remarque Ruth Amossy (1991 : 34), dans le texte littéraire, "une représentation traditionnelle peut exister et être reproduite sans qu'il soit nécessaire que l'auteur et ses lecteurs y croient effectivement ». L'on peut d'ailleurs remarquer que si les femmes peuvent déjouer les stéréotypes, les hommes ne témoignent guère d'une virilité incontestable. Comme les femmes, les hommes, loin d'être exemplaires, font preuve de nombreux défauts et sont caricaturés : «Quel idiot que le mâle !» (Pitié pour les femmes : 63), s'exclame Costals luimême. Les annonces matrimoniales au début des Jeunes Filles stigmatisent aussi bien les femmes que les hommes. Les deux extraits fonctionnent en miroir : la critique d'abord de la femme, puis de l'homme, tous deux avides de plaire, tous deux agissant par conformisme social. L'ironie de Montherlant s'exerce donc aussi contre l'homme et notamment contre sa cupidité : ainsi cet homme en quête d'une " âme sœur fortunée " (Les Jeunes Filles: 17), expression paradoxale chez cet homme pour qui l'appât du gain le dispute à la recherche de l'amour.

Quant au héros, Costals, il se montre rarement héroïque. C'est ainsi qu'il est parfois présenté comme un bavard prétentieux qui «dégoise» (Les Lépreuses: 26) ou « déblatère " (Les Jeunes Filles: 73). La critique a déjà mis en avant les limites et les faiblesses de ce dernier, notamment Patrick Brunel qui consacre un article aux "dépendances» du héros dans lequel il note que, par le rire, "c'est sa propre vulnérabilité que, plus ou moins consciemment, Costals cherche à travestir » (Brunel 1996 : 88). Il est ainsi possible de se demander si la maîtrise affichée par Costals ne cache pas une certaine vulnérabilité. Tout se passe en effet comme si la peur de ne pas correspondre au modèle héroïque qu'il se fixait poussait le héros à truquer en permanence son rapport aux femmes. Ainsi, s'il envisage de se marier avec Solange, ce ne peut être qu'à des conditions qui lui permettent de mettre fin à ce contrat sitôt qu'il en aura assez. Pour protéger sa "part essentielle ", Costals s'abrite derrière des idéaux virils qui lui servent de paravents et l'empêchent de s'ouvrir au risque (réel cette fois-ci et non plus simplement fantasmé) d'une relation authentique à l'autre. Dans une belle définition, la philosophe Maria Michela Marzano note qu'aimer « signifie partager son espace - l'espace de son corps, l'espace de sa parole, l'espace de ses silences - et faire le pari que l'autre accepte de partager le sien propre, sans nous déposséder du nôtre, sans le ravager, sans le quitter brutalement en le laissant vide» (Marzano 2007: 60). Or Costals refuse précisément cette réciprocité.

\section{L'amusement créateur}

Si Montherlant a pu présenter son roman comme un traité de morale destiné à réactiver les forces d'une nation endormie, il a également insisté sur sa dimension ludique en parlant d'« un livre composé de gags à la Charlot, un livre comique, ce que les gens n'ont peut-être pas vu " (Marchand 1980 : 38). Simone de Beauvoir n'envisage par exemple jamais le roman autrement que comme un ouvrage sérieux, un essai qui viserait à démontrer la faiblesse des femmes. Or si le roman peut se lire comme la satire d'un univers féminin, il peut aussi apparaître comme «le territoire du jeu et des hypothèses » (Kundera 1986 : 97). L'on constate en effet que les quatre caractéristiques 
discursives du sérieux identifiées par Jean-Marc Moura (2010:117) sont subverties dans le roman, qu'il s'agisse de la vocation performative, du refus de la polysémie, de la fiabilité du locuteur ou de la maîtrise du discours.

Le roman renonce d'abord en partie à sa vocation performative en exhibant son énonciation : les interventions de l'auteur que nous avons déjà indiquées contribuent à déstabiliser l'autorité du discours en brisant l'illusion discursive et littéraire. En outre, l'écrivain multiplie les références intertextuelles dans son roman au point de brouiller sa cohérence (vingt-sept références explicites dans le premier tome, trente-huit dans le deuxième, trente-neuf dans le troisième, soixante-douze dans le quatrième). L'écrivain mobilise ainsi des références de plus en plus nombreuses au fil de l'œuvre (jusqu'à la saturation dans le dernier tome) qui lui permettent de cultiver la discordance car elles relèvent de discours très divers, non seulement littéraires, mais aussi historiques, philosophiques, scientifiques ou populaires. Dans ce patchwork de citations, l'écrivain multiplie les références religieuses (Thérèse de Lisieux, L'Ecclésiaste, les Évangiles, Saint-Cyran) sans renoncer à citer fréquemment le prophète de la mort de Dieu (Nietzsche). Il mêle les références antiques (Juvénal, Catulle, Sénèque, Hésiode, Homère) et les références contemporaines (Paul Morand, Paul Claudel, André Gide, Paul Valéry, Colette), il renvoie à la culture savante (Malebranche, Descartes) aussi bien qu'à la culture populaire (les livres de la Bibliothèque rose, le magazine Cri-Cri). Il ruine ainsi les principes de transparence et d'univocité du discours sérieux.

27 La poétique romanesque ne se met pas non plus au service d'un message monologique. $\mathrm{Au}$ contraire, en multipliant les perspectives à travers des formes variées (lettres, récits à la troisième personne, carnets mais aussi annonces et articles de journaux), l'écrivain crée l'ambiguïté. En fonction du point de vue adopté, la vérité semble se métamorphoser. Le récit à la troisième personne donne par exemple à Andrée plus d'épaisseur qu'elle n'en a quelquefois dans ses lettres. À l'inverse, il permet de prendre de la distance avec Costals, qui semble dans ses articles prôner ouvertement une guerre des sexes, " avec des théories, pleines de cette fatigante habitude qu'il avait de tirer un trait entre les sexes " (Pitié pour les femmes : 89). Pierre Danger note avec justesse que « les instances narratives sont ainsi comme des pelures d'oignon: nous désespérons d'atteindre le cœur, car celui-ci n'est fait, au bout du compte, que de ces pelures successives qui le dérobent à notre attente » (Danger 1996: 65). Ainsi, la cohérence et la maîtrise de la composition sont à leur tour ébranlées au point que la progression du texte paraisse énigmatique. Moins guidé par une morale finale que par la seule fantaisie, le texte progresse dans un désordre apparent.

Enfin, alors que l'émetteur sérieux assume son discours en s'identifiant comme une source unique et responsable, l'humoriste peut se désolidariser de son texte. Or le roman de Montherlant cultive le brouillage de l'identité et de l'origine de la parole. Il convient sans doute de distinguer Montherlant auteur et Costals personnage là où Simone de Beauvoir les confond continuellement dans son article. Néanmoins, si Montherlant invite le lecteur à ne pas le confondre avec son héros dans l'avertissement précédant son roman, il n'en cultive pas moins là encore l'ambiguïté. En effet, son héros lui ressemble beaucoup : écrivain lui-même, obsédé par la décadence morale de la France et critique à l'égard d'une société qu'il juge efféminée. En même temps, le récit à la troisième personne présente le discours de Costals comme excessif, si bien qu'il paraît impossible de le prendre complètement au sérieux. 
Ainsi, le dispositif romanesque des Jeunes Filles permet de nuancer la misogynie prêtée à son auteur. Le roman vise peut-être moins à produire une leçon claire qu'à amuser le lecteur.

Si Montherlant n'a pas semé le "trouble dans le genre », pour reprendre le titre de l'essai de Judith Butler, du moins l'a-t-il semé dans la critique. Le roman a ainsi pu être reçu aussi bien comme un brûlot misogyne qui refléterait le mépris de l'auteur pour les femmes (c'est la lecture qu'en fait Simone de Beauvoir) que comme un «manuel de savoir-vivre à l'usage de la femme moderne » ainsi que le prétendit Sarah Vajda le jour de sa soutenance de lettres (Vajda $2012: 36$ ).

1 Il serait absurde de faire de Montherlant un militant de l'émancipation féminine dans la mesure où son œuvre se construit à partir d'un idéal viril qui consacre la supériorité du masculin sur le féminin. Le roman se présente bien à cet égard comme une satire qui vise à ridiculiser les femmes pour provoquer un sursaut dans la nation et lui permettre de retrouver force et lucidité.

2 Pour autant, faut-il complètement prendre au sérieux les déclarations misogynes qui émaillent le roman? Sans doute faut-il d'abord faire la part dans le roman de tout ce qui relève de la peinture d'une époque. En outre, la férocité de Montherlant s'explique par la perspective cynique qu'il adopte et qui le pousse à tout mettre en cause, les comportements des femmes aussi bien que ceux des hommes.

3 Si Montherlant construit un idéal viril qui repose sur la puissance sexuelle masculine, il n'en dessine pas moins un horizon possible d'émancipation pour les femmes. Sans doute, comme le déplore simone de Beauvoir, contribue-t-il dans le roman à essentialiser le féminin et le masculin, mais nul dans le roman n'est complètement prisonnier de son sexe. Les identités masculine et féminine circulent et transforment cette entreprise de démystification en jeu: «vous êtes toutes les mêmes; vous croyez toujours que je ne plaisante pas quand je plaisante, et que je plaisante quand je ne plaisante pas", indique Costals à Andrée (Pitié pour les femmes: 114). Le roman se construit en effet comme un jeu qui multiplie les formes, les points de vue et les références pour mieux provoquer l'amusement du lecteur.

Moins sérieuse qu'elle n'y paraît, la satire de la femme n'est donc peut-être pas à prendre au premier degré, Montherlant invitant d'abord les femmes à réagir contre un ordre imposé par les hommes, contre la caricature qu'on peut faire d'elles. Si le roman propose une morale, la leçon n'en est donc pas aussi claire qu'on pourrait le penser. L'«horreur du féminin » n'est peut-être qu'un versant de l'œuvre, sa face la plus visible, certes, mais comme souvent chez Montherlant la réversibilité est la règle: l'« horreur du féminin " n'exclut pas une certaine tendresse à l'égard des femmes, moins visible sans doute car aussi plus souterraine et en définitive peut-être plus vraie. Ainsi, dans l'œuvre de cet écrivain réputé misogyne, même les femmes semblent finalement trouver une place. 


\section{BIBLIOGRAPHIE}

Amossy, Ruth, 1991, Les Idées reçues : sémiologie du stéréotype, Paris, Nathan.

Arx, Paule d', 1995, Henry de Montherlant ou Les Chemins de l'exil, Paris, Librairie A.-G. Nizet.

Audouin-Rouzeau, Stéphane, 2011, « La Grande Guerre et l'histoire de la virilité », dans A. Corbin, J.-J. Courtine et G. Vigarello (dir.), Histoire de la virilité. Le triomphe de la virilité. Le XIXe siècle, Paris, Seuil.

Beauvoir, Simone de, [1949] 2008a, Le Deuxième Sexe, tome I : Les faits et les mythes, Paris, Gallimard. Beauvoir, Simone de, [1949] 2008b, Le Deuxième Sexe, tome II : L'expérience vécue, Paris, Gallimard. Blanc, André, 1995, L'Esthétique de Montherlant, Paris, SEDES.

Bourdieu, Pierre, 1998, La Domination masculine, Paris, Seuil.

Brunel, Patrick, 1996, « Les dépendances de Costals », Roman 20-50, n 21, p. 85-94.

Collin, Françoise, 1992, « Différence et différend », dans G. Duby et M. Perrot (dir.), Histoire des femmes en Occident, Le $\mathrm{XX}^{e}$ siècle, Paris, Plon.

Danger, Pierre, 1996, « Les Saintes Écritures », Roman 20-50, nº 21, p. 57-67.

Domenget, Jean-François, 2014, « Montherlant, le parti de la désinvolture », Le Magazine littéraire, $\mathrm{n}^{\circ} 541$, p. 56-57.

Drieu la Rochelle, Pierre, [1939] 1973, Gilles, Paris, Gallimard.

Kremer, Jean-Pierre, 1987, Le Désir dans l'œuvre de Montherlant, Paris, Archives des lettres modernes.

Kundera, Milan, 1986, L’Art du roman, Paris, Gallimard.

Lancrey-Javal, Romain, 2007, " Montherlant défenseur des femmes ", Journée Henry de Montherlant du 25 septembre 2007 à Bruxelles, Actes, à l'initiative du comte Henri de Meeûs, de Pierre Somville et de Pierre Duroisin, Bruxelles, Tropismes Librairies, [En ligne], http://www.montherlant.be/ audio-27-romain-lancrey-javal.html, consulté le 26 novembre 2019.

Marchand, Jean-José, 1980, Jean-José Marchand interroge Montherlant, Paris, Éditions Jean-Michel Place.

Marzano, Maria Michela, 2007, « Amour », dans M. M. Marzano (dir.), Dictionnaire du corps, Paris, PUF.

Montherlant, Henry de, [1920] 1963, La Relève du matin, Essais, Paris, Gallimard, coll. « Bibliothèque de la Pléiade».

Montherlant, Henry de, [1938] 1963, L'Équinoxe de septembre, Essais, Paris, Gallimard, coll. « Bibliothèque de la Pléiade».

Montherlant, Henry de, [1957] 1963, Carnets 1930-1944, Essais, Paris, Gallimard, coll. « Bibliothèque de la Pléiade ».

Montherlant, Henry de, 1972, La Marée du soir (Carnets 1968-1971), Paris, Gallimard.

Moura, Jean-Marc, 2010, Le Sens littéraire de l'humour, Paris, PUF. 
Sangars, Romaric, 2012, «Le Soleil en face. Montherlant, professeur d'énergie ", dans C. Dedet (dir.), Montherlant aujourd'hui, Paris, Éditions de Paris.

Sipriot, Pierre, 1979, Album Montherlant, Paris, Gallimard, coll. « Bibliothèque de la Pléiade ».

Vajda, Sarah, 2012, « Montherlant s'éloigne... revient », dans C. Dedet (dir.), Montherlant aujourd'hui, Paris, Éditions de Paris.

\section{Corpus}

Montherlant, Henry de, [1936] 1972, Les Jeunes Filles, Paris, Gallimard.

Montherlant, Henry de, [1936] 1972, Pitié pour les femmes, Paris, Gallimard.

Montherlant, Henry de, [1937] 1972, Le Démon du bien, Paris, Gallimard.

Montherlant, Henry de, [1939] 1972, Les Lépreuses, Paris, Gallimard.

\section{RÉSUMÉS}

C'est au cycle des Jeunes Filles (1936-1939) notamment que Henry de Montherlant doit sa réputation d'écrivain misogyne. Dans cette série de quatre romans, l'écrivain provoqua en effet le scandale en décrivant de jeunes femmes amoureuses gravitant autour du célèbre écrivain Pierre Costals. Le portrait qu'en fait Montherlant n'est guère flatteur. La féminité est présentée comme une gangrène qui n'oblitère pas seulement la santé du héros, mais aussi celle de la nation, présentée comme un corps viril qui risque d'être émasculé. Pourtant, le roman de Montherlant n'est pas complètement hostile aux femmes si l'on veut bien l'envisager comme un parcours ludique qui, à travers le tableau d'une guerre des sexes, déjoue les attentes de son lecteur en refusant les classifications trop simples.

It is to the collection of work Les Jeunes Filles (1936-1939) that Henry de Montherlant owes his reputation as a misogynist writer. In this series of four novels, the writer provoked scandal by recounting various stories of amorous women in the life of the famous writer Pierre Costals. The portrait that Montherlant paints of women is far from flattering. Femininity is presented as a gangrene that not only obliterates the protagonist's good health, but also that of the nation at large-in the novel, the nation is characterized as a living body that risks emasculation to the feminine. Montherlant's novel is not, however, completely hostile to women if one decides to interpret it as a playful journey instead; one that traverses the divisive realm of the war of the sexes and defeats its reader's expectations by refuting oversimplified classifications.

\section{INDEX}

Keywords : masculinity, emasculation, body, French nation, misogyny, Montherlant (Henry de) Mots-clés : virilité, émasculation, corps, nation française, misogynie, Montherlant (Henry de)

\section{AUTEUR \\ PIERRE DAMAMME}

Sorbonne Université, CELLF (UMR 8599) 